\title{
Cognitive orientations in marathon running and "hitting the wall"
}

\author{
Clare D Stevinson, Stuart J H Biddle
}

\begin{abstract}
Objectives-To investigate whether runners' cognitions during a marathon are related to "hitting the wall". To test a new and more comprehensive system for classifying cognition of marathon runners.

Methods-Non-elite runners $(\mathrm{n}=66)$ completed a questionnaire after finishing the 1996 London marathon. The runners were recruited through the charity SPARKS for whom they were raising money by running in the race.

Results-Most runners reported that during the race their thoughts were internally associative, with internally dissociative thoughts being the least prevalent. Runners who "hit the wall" used more internal dissociation than other runners, indicating that it is a hazardous strategy, probably because sensory feedback is blocked. However, internal association was related to an earlier onset of "the wall", suggesting that too much attention on physical symptoms may magnifiy them, thereby exaggerating any discomfort. External dissociation was related to a later onset, probably because it may provide a degree of distraction but keeps attention on the race.
\end{abstract}

Conclusions-"Hitting the wall" for recreational non-elite marathon runners is associated with their thought patterns during the race. In particular, "the wall" is associated with internal dissociation.

(Br F Sports Med 1998;32:229-235)

Keywords: cognitive strategies; marathon running; "the wall”

Most research into the cognitions of marathon runners is based on the concepts of association and dissociation identified by Morgan and Pollock. ${ }^{1}$ An associative strategy involves keeping attention focused on bodily sensations and/or factors critical to performing the task, while a dissociative one attempts to avoid thinking about such cues. Put simply, association equates to concentration on a task, while dissociation means distraction from it.

Several studies have investigated how these C D Stevinson S J H Biddle

Correspondence to: Professor S Biddle, Department of PE, Sports Science and Recreation Management, Loughborough University, Loughborough, Leics LE11 3TU, United Kingdom

Accepted for publication 26 February 1998 symptoms from blisters, muscle strains, and
cramp to dehydration, stress fractures, and heat stroke. One could argue that an associative ing attention from physical sensations, any warning signals of injury or pain are masked, putting the runner at risk of a range of painful strategy would avoid these problems because careful monitoring of bodily sensations would detect early indications of trouble, allowing the necessary steps to be taken to prevent the problem developing-for instance, drinking more fluid, stretching, applying Vaseline, dropping out. However, Masters and Lambert ${ }^{3}$ have rejected this idea. All the runners in their sample who had to drop out of a marathon because of injury were associating before noticing the injury.

As well as being a causal contributor of pain, Morgan $^{2}$ also claimed that dissociation was a response to it. When interviewing non-elite runners of the Boston marathon, he found that they all made reference to "hitting the wall" during the race and dealing with it by thinking about something else to distract themselves from the pain.

"The wall" is a term used in the context of a marathon to refer to the point, generally at about 20 miles, where glycogen supplies have been exhausted and energy has to be converted from fat. This is a slower process than with glycogen and the consequential shortage of glucose to the brain may result in hypoglycaemia, the scientific term for "the wall". This can be an extremely unpleasant experience with symptoms including a lack of physical coordination, dehydration, paraesthesia (tingling or numbness in the toes or fingers), nausea, muscle spasms, dizziness, an inability to think clearly, and extreme physical weakness.

Morgan $^{2}$ also interviewed elite marathon runners who, in contrast with the Boston runners, described "the wall" as a myth. They insisted that by associating with one's body and adjusting the pace, the symptoms of "the wall" could be avoided. The non-elite marathon participants in the study of Morgan ${ }^{2}$ implied that "hitting the wall" was an inevitable experience that could only be endured by dissociating oneself from the pain. However, the message from the elite runners was that associating with sensory signals ensured that "the wall" could be avoided. No support for either of these positions exists from other studies.

Summers et $a l^{4}$ found that $56 \%$ of their nonelite sample reported "hitting the wall", but there was no relation to the type of cognitive strategy employed. Both Okwumabua ${ }^{5}$ and Masters and Lambert ${ }^{3}$ concluded that dissociation does not occur in response to "the wall", because there was less dissociation after the 20 mile mark than before it.

One of the problems with both these studies is that neither Okwumabua ${ }^{5}$ nor Masters and Lambert ${ }^{3}$ actually asked runners if they "hit the wall". They simply based their conclusions on 
the knowledge that most people who do hit it do so after about 20 miles. Investigators must directly address the issue of "hitting the wall" if they are to attempt to understand whether cognitive strategy can have a role in avoiding it, or at least coping with it.

METHODOLOGICAL ISSUES

Other problems with studies of cognitive strategies concern the different ways in which data are collected and cognitions classified. There is no easy way to find out what people are thinking while they are running. Morgan ${ }^{2}$ carried out interviews with his participants after the event. Post-race questionnaires were used by several investigators. ${ }^{34}$ Schomer $^{6}$ believed that this form of retrospective self report data would be inaccurate because of poor recall or deliberate distortion. $\mathrm{He}$ attached micro cassette recorders to his participants and asked them to say aloud everything that came into their minds as they ran. However, it is argued that this intrusive method is much less likely to produce reliable data than a retrospective approach. Participants may feel self conscious about reporting certain thoughts, or find some too abstract or brief to put into words. Equally, they may wish to save their energy for running rather than talking aloud. Having to verbalise one's stream of consciousness may affect what is actually thought about, and there is potential for a social desirability effect, with runners trying to provide responses for the researchers that they think they are searching for. Asking runners to recall their thoughts retrospectively avoids the major problem of interference, and is likely to produce more data than in the aroused breathless state of running.

Summers et $a l^{4}$ and Masters and Lambert ${ }^{3}$ tried to measure the extent to which each cognitive strategy was employed by asking runners to write down all the things they thought about during the race, then classifying each thought as associative or dissociative and transforming the number of statements into a proportion. There are various problems with this free recall method of quantifying cognitions. Attempting to list every little thought that entered their heads may seem overly tedious, difficult, or embarrassing to participants. They may have forgotten much, or only remember the first or last few miles, showing a primacy or recency effect. Some people may produce very detailed specific lists, while others write down only broad themes that encompass various thoughts, such as their family. All these factors can have a marked effect on frequency counts, so that it is impossible to know how accurately they represent the prevalence of association and dissociation. In addition, there is a risk of ambiguously worded thoughts being wrongly classified. Although raters are trained and inter-rater reliability is measured to reduce the risks of misclassification, essentially what is being recorded is the rater's interpretation of what the runner wrote down. An accurate interpretation therefore depends on the runners conveying very clearly and specifically what they were thinking.
In considering these issues it is suggested that the best way of producing data that accurately represent runners' thoughts is to put the onus on the runners themselves. Since they presumably know what they were thinking, the runners are in the best position to label their thoughts as associative or dissociative and make a relative estimate about how much of the race was spent in each type of thought. In order for them to do this, they must completely understand what is meant by the terms association and dissociation, and so precise definitions and various examples need to be provided. Unlike the free recall method, thoughts do not have to be expressed as participants carry out the analysis in their heads, and so the problem of personal thoughts not being analysed because of embarrassment is eliminated.

Classification of cognitions is also problematic because of the slightly different ways in which association and dissociation have been operationalised. For Morgan, ${ }^{2}$ association simply meant attending to bodily sensations, while dissociation meant distraction from them. However, Sacks et $a l^{l}$ and Masters and Lambert $^{3}$ defined the terms more widely so that association included monitoring both internal states and factors critical to performing the task, and dissociation referred to distraction from both of these types of cues.

The difference between these definitions is important since it affects whether particular thoughts are labelled associative or dissociative. During a marathon, attention on mile split times is very much performance related and therefore associative. However, if association only includes attending to physical symptoms, then thinking about split times would be classed as dissociative, in that it is a distraction from discomfort. Similarly in the study of Clingman and Hilliard, ${ }^{8}$ the associative strategies of race walkers concerned technique, with walkers focusing on either stride length or cadence. Since the concentration on technique distracts from the pain of exertion, this might also be considered a dissociative strategy.

As well as definitions of the cognitive strategies being inconsistent between studies, so too are the terms used. Pennebaker and Lightner ${ }^{9}$ and Padgett and Hill $^{10}$ used the terms internal focus (bodily sensations) and external focus (environmental stimuli) instead of association and dissociation in their manipulations of cognitive strategy.

Acevedo et $\mathrm{l}^{11}$ used the same terms in trying to classify the cognitions of ultra-marathoners, but for two reasons this internal/external focus distinction was inappropriate. Firstly, a simple classification based purely on direction of attentional focus is not sophisticated enough to cover the vast range of thoughts that may pass through a runner's mind during a 100 mile race. Internal focus refers only to bodily sensations, excluding any performance related thoughts based on external information-for example, split times, distance markers. Similarly, external focus accounts for attention being on environmental cues, but precludes thoughts of an imaginary fantasy nature. It is 
the use of the external focus label that provokes the second criticism of the terminology of Acevedo et al. ${ }^{11}$ The examples they offered for an external focus were music melodies, problems at work, maths problems, and sex. Far from being external cues, these imaginary type thoughts all require attention to be directed inwardly, so ambiguously, the external focus category did not refer to external stimuli at all. Presumably Acevedo et $a l^{11}$ intended their external focus category to simply represent distraction from internal sensations, but their operationalisation of the terms internal/ external focus resulted in an oversimplistic, ambiguous, and inappropriate way of classifying cognitions.

Padgett and Hill ${ }^{10}$ acknowledged the difference between the imaginary kind of dissociation and the external stimuli type, by having two categories of dissociation techniques. One was called dissociation and involved hearing imaginary songs and composing letters. The other was labelled external focus and required participants to count the number of hurdles on the track and cars in the car park, as they ran laps.

Goode and Roth ${ }^{12}$ also recognised that there are two types of dissociative thoughts, those of an imaginary/daydreaming nature and those involving external stimuli. Their factor analysis of runners' thoughts did not, however, distinguish between different types of associative thought. Items relating to the monitoring of bodily responses and the demands of the running activity were all loaded on to one associative factor. However, it is worth noting that the data of Goode and Roth ${ }^{12}$ were gathered from runners during training runs. According to Morgan et al, ${ }^{13}{ }^{14}$ runners do significantly less associative thinking when training than when racing, when the demands of the activity are tougher and concentration is required for maximal effort and optimal performance.

Schomer ${ }^{6}$ attempted to avoid the confusion over what is meant by association and dissociation by using the terms task related and task unrelated. Associative thoughts therefore included any that were related to the task-for instance, bodily sensations, conditions, pacewhile dissociative thoughts incorporated those that were not connected to the task-for instance, daydreaming, problem solving, admiring the view. However, Summers et al used similar definitions and reported that $63 \%$ of listed thoughts could not be classified because they were associative in the sense of being related to the task but dissociative in distracting from the discomfort of running. Examples included talking to other runners and setting subgoals of running from one drinks station to the next.

This shows that a dichotomous association versus dissociation distinction is too simplistic for categorising runners' thoughts and that there is a need for a more comprehensive classification system.

PURPOSES OF THE STUDY

The major aim of this study was to examine the relation between the cognitions of runners during a marathon and their experiences of "hitting the wall". The study also addressed some of the methodological issues that have been raised in other studies of cognitive strategies and tested a more comprehensive system for classifying cognitions of marathon runners.

\section{Method}

SAMPLE

Participants were entrants in the 1996 London marathon. They had been offered guaranteed places in the race through their association with the charity SPARKS (SPort Aiding medical Research for KidS). Runners from the SPARKS team (about 400) were randomly selected $(n=100)$ and sent a letter informing them of the study and inviting them to indicate if they did not wish to be involved. None did so, and all 100 runners were mailed a questionnaire and an accompanying letter from the senior investigator, which were received eight days before the race. The letter introduced the nature of the project, assured respondents that all information would be treated with confidence, and provided instructions on the completion and return of the questionnaire.

Seventy four of the 100 questionnaires were returned, of which eight had to be discarded because of incomplete data or wrongly completed questions. The final sample therefore consisted of 66 participants (56 men and 10 women). The mean age was 36.11 years ranging from 21 to 59 years. The mean number of previous marathons run was 1.39 (range $0-10$ ), and the sample included 35 first timers. The mean finishing time was four hours 16 minutes (range three hours 00 minutes to six hours 28 minutes). All runners were slower than two hours 45 minutes so were classed as non-elite runners, since the Amateur Athletics Association recognises times faster than 2.45 as elite performances.

\section{RESEARCH INSTRUMENT}

A new classification system was developed for identifying thoughts as associative or dissociative, involving two dimensions; the first was task relevance. Any thoughts that were relevant to task performance-for example, monitoring internal state, calculating mile splits-were considered associative. Thoughts that were not directly relevant to task performance, although they may or may not be connected with the task-for example, daydreaming, observing scenery or spectators-were labelled dissociative. The second dimension was direction of attention. Task relevant thoughts involving an internal focus-for example, physical sensations-were classified as internal association, task relevant thoughts involving an external focus - for example, split times-were classified as external association. Similarly, task irrelevant thoughts with an internal focus-for example, daydreams-were categorised as internal dissociation, while task irrelevant thoughts with an external focus-for example, scenery-were categorised as external dissociation. A pilot study in which 10 runners were asked to categorise a list of thoughts confirmed 
Table 1 Extent of usage of cognitive orientations during the race

\begin{tabular}{llllc}
\hline Rank & Cognitive orientation & Mean & SD & \% of race \\
\hline 1 & Inward monitoring $^{1}$ & 2.95 & 1.33 & 36.88 \\
$2=$ & Outward monitoring $^{2}$ & 2.27 & 1.16 & 28.38 \\
$2=$ & Outward distraction $^{3}$ & 2.09 & 1.16 & 26.13 \\
4 & Inward distraction $^{123}$ & 0.67 & 0.87 & 8.38 \\
\hline
\end{tabular}

Numbered superscripts indicate significant differences $(p<0.05)$ between cognitive orientations when the numbers match.

Table 2 Cognitive orientations of runners who "hit the wall" and those who did not

\begin{tabular}{llll}
\hline Cognitive orientation & & Hit wall & Did not \\
\hline Inward monitoring & Mean & 3.00 & 2.90 \\
& SD & 1.33 & 1.35 \\
Outward monitoring & Mean & 2.11 & 2.45 \\
& SD & 0.99 & 1.31 \\
Outward distraction & Mean & 1.97 & 2.23 \\
& SD & 1.12 & 1.20 \\
Inward distraction & Mean & 0.89 & $0.42^{\star}$ \\
& SD & 1.02 & 0.56 \\
\hline
\end{tabular}

*Denotes significant difference at the $\mathrm{p}<0.05$ level.

that these four categories were all inclusive and mutually exclusive.

The questionnaire to participants provided them with a definition and examples for each category and asked them to give each category a rating, from 0 to 8 , to represent the approximate proportion of the race spent engaged in those types of thoughts, with the four ratings totalling 8 to represent the whole race. The scale was represented by the wording "no time at all" (0), "about half the time" (4), and "all the time" (8). Runners were clearly instructed to ensure that their four ratings added up to 8 to represent all of the race period. The exact instructions to participants were as follows.

People think about a variety of things while running a marathon. These thoughts can be one of four types, which are described below in the categories A, B, C, and D. In the spaces provided, please give each of the four categories a rating from 0 to 8 using the scale below, to indicate approximately how much of the race was spent in those types of thoughts. The total of your scores over the four categories must equal 8. There is an example provided to help you. The definitions and examples of the four categories are as follows: $(A)$ inward monitoring: attention is focused inwardly on how your body feels while running - for example, breathing, muscle soreness, thirst, fatigue, perspiration, blisters, nausea; (B) outward monitoring: attention is focused outwardly on things important

Table 3 Onset, duration, and finish point of "the wall" (in miles)

\begin{tabular}{lrrr}
\hline & Mean & $S D$ & Range \\
\hline Onset & 18.69 & 2.74 & $12-25$ \\
Duration & 4.91 & 3.05 & $1-14$ \\
Finish & 23.31 & 2.86 & $16-26$
\end{tabular}

Table 4 Number of men and women "hitting the wall"

\begin{tabular}{llcl}
\hline & Men & Women & $\begin{array}{l}\text { Total No } \\
\text { (\%) }\end{array}$ \\
\hline Hit wall & 33 & 2 & $35(53)$ \\
Did not & 23 & 8 & $31(47)$ \\
Total (No) & $56(85)$ & $10(15)$ & \\
\hline
\end{tabular}

to performing the task-for example, strategy, mile markers, water stations, split times, route, conditions; (C) inward distraction: attention is focused inwardly on anything irrelevant to the task-for example, daydreams, imagining music, maths puzzles, philosophy, religion; (D) outward distraction: attention is focused outwardly on things unimportant to task performance-for example, scenery, spectators, other runners, environment.

Participants were also asked to record their finishing time and state whether or not they "hit the wall". If they did, they were also asked to indicate the point (in miles) at which they hit it and for how many miles the symptoms lasted.

\section{Results}

\section{COGNITIVE ORIENTATIONS}

Table 1 displays the mean scores and standard deviations for each cognitive orientation and also the percentage of the race that was taken up by each type. Paired sample $t$ tests were used to identify any significant differences between the means. The mean for inward monitoring was significantly higher than for the other three categories. There was no difference between outward monitoring and outward distraction, and the inward distraction mean was lower than the other three strategies.

\section{MARATHONER'S WALL}

Thirty five of the 66 runners (53\%) reported that they "hit the wall" during the race. The remaining $31(47 \%)$ did not hit it, so two discrete groups existed. Table 2 shows the mean scores of each cognitive orientation for the two groups. $t$ tests showed that inward distraction scores were significantly greater for those who "hit the wall" than those who did not $(p<0.05)$. Information was also gathered from the runners who reported "hitting the wall" with regard to the point at which it started and how long it lasted. Table 3 shows the means, standard deviations and ranges of the onset point of "the wall", and its duration and finish point, all in miles.

Product-moment correlation coefficients showed a significant positive relation between the onset of "the wall" and its duration $(r=$ $0.51, \mathrm{p}<0.05)$ and a significant negative correlation between onset and finishing time $(r=$ $-0.51, \mathrm{p}<0.05)$. Furthermore, there was a significant negative relation between the amount of inward monitoring during the race and the onset of "the wall" $(r=-0.39, \mathrm{p}<0.05)$, and a significant positive relation between inward monitoring and the duration of "the wall" $(r=$ $0.46, \mathrm{p}<0.05)$. There was also a positive correlation between outward distraction and onset $(r$ $=0.56, \mathrm{p}<0.05)$.

The only gender difference in this study concerned "hitting the wall". Table 4 shows the number of men and women that reported either "hitting the wall" or not doing so. $\chi^{2}$ analysis showed that hitting the wall was associated with men significantly more than women $\left(\chi^{2}=5.16, \mathrm{p}<0.05\right)$. 


\section{Discussion}

COGNITIVE ORIENTATIONS

As far as a runner's cognitions were concerned, the most popular thought category was inward monitoring. More time was spent on attention to physical sensations than any other kind of stimulus. Outward monitoring and outward distraction were the next most common types of thought, with inward distraction being used significantly less than the other categories.

The significant difference between the amount of inward and outward monitoring, and similarly between inward and outward distraction, appears to justify the subdivision of association and dissociation by the direction of attentional focus. Rather than refer to association in general, the results showed that much more attention was given to physical sensations than tactical aspects of the race. With dissociation, focusing on environmental stimuli was far more popular than imaginary type thoughts. Outward monitoring and outward distraction were equally prevalent and, put together, indicate that over half the race was spent with attention focused externally on both task relevant and task irrelevant stimuli.

The popularity of inward monitoring supports the findings of Masters and Lambert, ${ }^{3}$ Okwumabua, ${ }^{5}$ and Summers et $a l,{ }^{4}$ who all reported a preference for association. Like the sample in the study of Summers et al, ${ }^{4}$ the runners were non-elite. The assertion of Morgan $^{2}$ that non-elite athletes tend to dissociate in races is not supported.

MARATHONER'S WALL

Just over half the sample reported "hitting the wall" during the race, which is similar to the proportion in the study by Summers et $a l^{4}$ That study found no relation between cognitive strategy and "hitting the wall", but the results of the present study yield some key findings on runners' attentional focus and their experience of "the wall".

Inward distraction proved to be far more prevalent for those runners who "hit the wall" than those who did not. This supports the theory of Morgan $^{2}$ that dissociation is a hazardous strategy. It is likely that being distracted from sensory signals and important aspects of the task meant that runners were not able to judge their pace very well and failed to stay fully hydrated, contributing greatly to "hitting the wall". However, it is apparently necessary to distinguish between the different types of dissociation. It was only inward distraction that differed between those "hitting the wall" and those not doing so. The amount of outward distraction was the same. It is possible that this was because focusing attention on environmental stimuli unintentionally helped to regulate pace without actually concentrating on it. Although it was distracting from the actual task of running, noticing spectators, aspects of the scenery, or, in particular, other runners, made runners inadvertently aware of the speed at which they were running as they passed by them or were overtaken.

Neither internal nor external association differed between runners "hitting the wall" and those not doing so. This contradicts the theory that association allows injury or pain to be avoided. Those who "hit the wall" were associating just as much as those who did not, so evidently association does not necessarily protect runners from "the wall". This is consistent with the results of Masters and Lambert, ${ }^{3}$ who found that association did not prevent runners from dropping out through injury.

Since inward distraction was related to "the wall", it would appear to play quite a significant role. The cognitive orientations of the 35 runners who "hit the wall" followed the same pattern of usage as those who did not hit it, and the sample as a whole. Inward monitoring was the most prevalent type of thought and inward distraction was the least. So it was not a case of people who "hit the wall" using more inward distraction than other types of cognition. Quite simply they made more use of it than runners who did not "hit the wall", which implies that it can have quite an adverse effect without being a major focus of attention.

Although the amount of association did not differentiate between those who "hit the wall" and those who did not, an examination of the cognitive orientations of just the runners who "hit the wall" showed that it was related to the point at which "the wall" started and how long it lasted. Greater inward monitoring was associated with an earlier onset and also a longer duration of the wall. This suggests that paying too much attention to the body made the runner magnify discomfort, so the symptoms of "the wall" were noticed earlier and for longer than if attention was not focused so closely on the body. In support of this, greater outward distraction was related to a later onset of "the wall". This is consistent with the explanation of Pennebaker and Lightner ${ }^{8}$ that a degree of distraction from bodily sensations reduced the degree to which they were encoded, so painful symptoms were not noticed as readily as when associating with them.

GENDER DIFFERENCE

Although no gender differences were found in cognitive orientations, significantly more men reported "hitting the wall" than women. Since women only represented $15 \%$ of the sample, this finding must be viewed with caution, but it does provide support for the view that women are more suited to the demands of endurance events such as the marathon. According to Blue, ${ }^{15}$ certain characteristics of the female metabolism mean that women suffer less than men in the later stages of a marathon and less often come up against "marathoner's wall". The American Academy of Orthopaedic Surgeons ${ }^{16}$ reported that women may be at an advantage in marathon and ultradistance events because their bodies convert to fatty acid metabolism more easily than those of men.

It may be that psychological factors rather than physiological ones could explain this apparent sex difference. Possibilities are that women are better at judging their pace so they manage to maintain a sensible rate that enable them to avoid "the wall", or that women train 
more thoroughly for the event, so their bodies are more prepared for the glycogen to fat conversion. This study provided no data that could be used to support any of these suggestions, so no conclusions can be made about why women are less likely to "hit the wall" than men.

\section{RECOMMENDATIONS TO RUNNERS}

One of the keys to completing the marathon in comfort and producing an optimum performance is to avoid "hitting the wall". This is not only a very unpleasant and painful experience but is related to slower finishing times. Avoiding it relies primarily on sufficient endurance training and stores of glycogen in the muscles. However, during the race, other factors, such as correct pace judgment and staying hydrated, are important. These are factors that can be affected by attentional focus, so it is about these that the following advice is given for non-elite runners of marathons.

Inward distraction should be avoided during the race. Total distraction from the task is hazardous because it blocks awareness of pace and warning signals from the body, so there is an increased danger of running too fast in the early stages when the body is fresh and hence suffering later when "the wall" is hit. For recreational runners, this may cause the race's special atmosphere to be missed. The atmosphere may well be one of the reasons why the experience is considered enjoyable.

Inward monitoring is therefore important for maintaining awareness of physical condition and allowing pace to be altered or more fluid to be taken in as appropriate to maximise comfort and performance. However, excessive attention to the body is inadvisable as this magnifies or exaggerates any discomfort, causing an earlier and longer encounter with "the wall". It is best therefore to make only brief but regular checks on bodily parts, rather than constant monitoring.

Most attention should be focused externally. Outward distraction allows the enjoyment derived from the atmosphere to be fully appreciated, and this degree of distraction minimises the effect of any discomfort while maintaining awareness of critical race related factors, so delaying the onset of "the wall". Similarly, outward monitoring keeps attention on the task but away from the discomfort, and is important for maximising performance.

FURTHER RESEARCH ISSUES

One issue that has yet to be addressed by research into runners' cognitive orientations is whether thoughts reflect a deliberate attempt to focus on certain things, or naturally occur in response to particular stimuli. Stating that runners "prefer" associating during races implies that they consciously choose to do so. However, there have been no attempts to investigate whether this is the case. The prevalence of inward monitoring may reflect a deliberate attempt to attend to physical sensations. Alternatively, feelings of discomfort may be so severe that they demand attention even though runners may attempt to distract themselves from them. Insights into the mind of the marathoner would be greatly enhanced by examining the role of runners' intentions with regard to their cognitions.

On a similar theme, there is scope for investigating the deliberate use of cognitive coping strategies in response to "the wall". Some of the non-elite runners in the study of Morgan ${ }^{2}$ reported specific mental rituals to help them deal with the pain of "the wall". The use of associative or dissociative strategies to increase pain tolerance in this kind of situation merits further attention by researchers.

1 Morgan WP, Pollock ML. Psychological characterisation of the elite distance runner. Ann N Y Acad Sci 1977;301:382403.

2 Morgan WP. The mind of the marathoner. Psychology Today 1978;11:38-49.

3 Masters KS, Lambert MJ. The relations between cognitive coping strategies, reasons for running, injury and performance of marathon runners. fournal of Sport and Exercise Psychology 1989;11:161-70.

4 Summers JJ, Sargent GI, Levey AJ, et al. Middle-aged, nonlite marathon runners: a profile. Percept Mot Skills 1982;54:963-9.

5 Okwumabua TM. Psychological and physical contributions to marathon performance: an exploratory investigation. fournal of Sport Behavior 1985;8:163-71.

6 Schomer HH. Mental strategies and the perception of effort of marathon runners. International fournal of Sport Psychology 1986;17:41-59.

7 Sacks MH, Milvoy P, Perry SW, et al. Mental status and psychological coping during a 100 -mile race. In: MH Sacks, chological coping during a 100 -mile race. In: MH Sacks, Human Kinetics Publishers, 1981:166-75.

8 Clingman JM, Hilliard VD. Race walkers quicken their step by tuning in, not stepping out. The Sport Psychologist 1990; 4:25-32.

9 Pennebaker JW, Lightner JW. Competition of internal and external information in an exercise setting. 7 Pers Soc Psychol 1980;39:165-74.

10 Padgett VK, Hill AK. Maximising athletic performance in endurance events: a comparison of cognitive strategies. fournal of Applied Social Psychology 1989;19:331-40.

11 Acevedo EO, Dzewaltowski DA, Gill DL, et al. Cognitive Acevedo EO, Dzewaltowski DA, Gill DL, et al. Cognitive
orientations of ultramarathoners. The Sport Psychologist 1992;6:242-52.

12 Goode KT, Roth DL. Factor analysis of cognitions during running: association with mood change. fournal of Sport and Exercise Psychology 1993;15:375-89.

13 Morgan WP, O'Conner PJ, Sparling PB, et al. Psychological characteristics of elite female distance runners. Int $\mathcal{F}$ Sports Med 1987;8:124-31.

14 Morgan WP, O'Conner PJ, Ellickson KA, et al. Personality structure, mood states and performance in elite male distance runners. International fournal of Sport Psychology 1988;19:247-63.

15 Blue A. Faster, higher, further: women's triumphs and disasters at the Olympics. London: Virago Press, 1988.

16 American Academy of Orthopaedic Surgeons. Athletic training and sports medicine. Park Ridge, Illinois: American Academy of Orthopaedic Surgeons, 1991.

\section{Commentary}

This study examines non-elite marathon runners' cognitions during a race in relation to "hitting the wall". Since Morgan and Pollock's seminal work in this area, a number of studies have examined the efficacy of associative and dissociative techniques for coping with stress of endurance events. However, such studies have shown mixed results, although the evidence appears to suggest that elite marathon runners prefer to use associative strategies. Nevertheless, the present article by Stevinson and Biddle addresses some unresolved issues in the literature. Cognisant of the difficulties imposed by available research instruments, the authors devise a new classification system which involves two dimensions (associative-dissociative; internal-external). Such a system allows four categories of response as opposed to the traditional two. Results suggest that only inward distraction separates 
non-elite runners who "hit the wall" from those not doing so. The study is important for two reasons. Firstly, it has addressed both methodological and theoretical issues raised in the research literature. Secondly, it has obvious clinical relevance in providing evidence to support practical recommendations for marathon runners which encourage the use of a combination of outward distraction and regular inward monitoring.

D SCULLY

\section{Ticks: danger lurking in the long grass}

Tick borne encephalitis is a meningoencephalitis caused by a flavivirus transmitted to humans by the bite of an infected tick for which a case fatality of around $1 \%$ has been reported. Eastern Europe, in particular western Hungary and the eastern part of Austria, are areas where tick borne encephalitis is a potential health problem. It is also present in Sweden, where $10-15 \%$ of adults with the illness are reported to develop paresis during the acute phase, and recovery may be slow. Ticks are most active in spring and summer, especially in forested areas where there is heavy undergrowth. Immunisation is available on a named patient basis using inactivated whole cell virus vaccine containing a suspension of purified tick borne encephalitis virus grown in chick embryo cells and inactivated with formalin. Immunisation was recommended for all those travelling to the pre-World Orienteering Championships in 1982 in Hungary and the World Championships held there in 1983, and was given to all the British team members with one exception who opted not to receive it. Immunisation was also advised

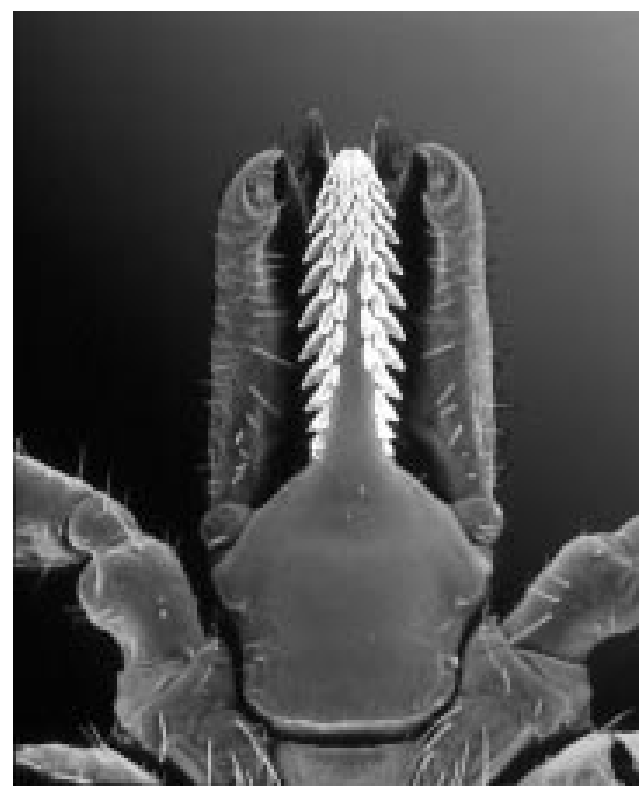

Figure 1 Scanning electron micrograph of the head and mouthparts of the female deer tick Ixodes scapularis. At the centre is the barbed skin-piercing blood-sucking mouthpart; at the lower centre is the head. Original magnification $\times 50$. for more recent orienteering events in Hungary and for those on walking holidays.

The risk of contracting tick borne encephalitis is not known, but there have been no reported cases among British competitors to date. A previous Hungarian world orienteering champion is now permanently disabled as a consequence of tick borne encephalitis. To minimise exposure to ticks, full body cover is recommended and competitors are encouraged to examine themselves after competition and to remove any ticks as soon as possible.

Lyme disease (caused by the spirochete, Borrelia burgdorferi) is the other tick borne condition that may be a problem. This condition was first described in the 1970s, with cases reported around the New Forest area and in parts of Scotland, although originally described in a small group of children in Old Lyme, Connecticut, USA. It presents a migratory rash (erythema migrans) and systemic involvement of heart, nervous system, and joints. There may be chronic persistent inflammation of the nervous system, joints, and skin if the condition is not treated. If recognised, it responds well to antibiotic therapy. A particular forest well known for ticks is Darnaway, used for the World Orienteering Championships in Scotland in 1976 - that is, before the first published descriptions of Lyme disease. With the 1999 World Championships in orienteering again being held in Scotland, the risk may need to be considered more seriously. Other tick borne diseases occur in North America and other parts of the world, but would be a rare problem in British sportspeople.

Ticks become attached by the back slanting serrations on the harpoon-like hypostome which becomes implanted in the skin (fig 1). A useful tick remover is available from Canac Pet Products (Becks Mill, Westbury Leigh, Westbury, Wiltshire BA13 3SD, United Kingdom, Tel: 01378-864775; current price $£ 5.99$ + small postal charge). It consists of a sophisticated pair of tweezers, which allow the body of the tick to be held while it is rotated in either a clockwise or anti-clockwise direction until the mouth parts release their hold from the host. It is very successful in removing the whole of the tick. Lyme disease is a possibility in the sportsperson who has been exposed to ticks and should be considered if they present with a skin rash, joint pain, or neurological features. After travel to central and eastern Europe or Scandinavia and in the presence of neurological features, tick borne encephalitis should be considered.

W N DODDS 